\title{
Neurology"
}

The most widely read and highly cited peer-reviewed neurology journal

The Official Journal of the American Academy of Neurology

\section{Prevalence of MRI abnormalities in people with epilepsy in rural China}

Indran Davagnanam, $\mathrm{FRCR}^{* 1}$, Zhibin Chen, $\mathrm{PhD}^{* 2,3,4}$, Chandrashekar Hoskote, FRCR ${ }^{5}$, Ding Ding, $\mathrm{MD}, \mathrm{PhD}^{6}$, Bin Yang, $\mathrm{MD}^{8}$, Yingli Wang, $\mathrm{MD}^{9}$, Taiping Wang, $\mathrm{MD}^{10}$, Wenling $\mathrm{Li}, \mathrm{MD}^{11}$, John S. Duncan, DM FRCP ${ }^{12}$, Wenzhi Wang, MD ${ }^{7}$, Josemir W. Sander, PhD FRCP $^{12,13}$, Patrick Kwan, PhD FRACP ${ }^{3,4}$

1. Academic Department of Neuroradiology, Department of Brain Repair and Rehabilitation, UCL Queen Square Institute of Neurology, Queen Square, London WC1N 3BG, UK

2. Clinical Epidemiology, School of Public Health and Preventive Medicine, Monash University, Melbourne, Australia

3. Department of Neuroscience, Central Clinical School, Monash University, Melbourne, Australia

4. Department of Medicine - Royal Melbourne Hospital, The University of Melbourne, Parkville, Australia

5. Lysholm Department of Neuroradiology, National Hospital for Neurology \& Neurosurgery, Queen Square, London WC1N 3BG, UK

6. Institute of Neurology, Huashan Hospital, Fudan University, Shanghai, China

7. Beijing Neurosurgical Institute, Beijing, China

8. Jiaozuo People's Hospital, Henan Province, China

9. Ningxia Medical University, Ningxia Province, China

10. Jincheng Emergency Medical Rescue Center, Shanxi Province, China

11. Affiliated Second Hospital, Hebei Medical University, Hebei Province, China

12. NIHR University College London Hospitals, Biomedical Research Centre, UCL Queen Square Institute of Neurology, Queen Square, London WC1N 3BG \& Chalfont Centre for Epilepsy, Chalfont St Peter SL9 0RJ, UK

13. Stichting Epilepsie Instellingen Nederland (SEIN), 2103SW Heemstede, Netherlands *These authors contributed equally to the manuscript.

Neurology® Published Ahead of Print articles have been peer reviewed and accepted for publication. This manuscript will be published in its final form after copyediting, page composition, and review of proofs. Errors that could affect the content may be corrected during these processes. Videos, if applicable, will be available when the article is published in its final form. 
Correspondence to: Prof. Kwan, Email: patrick.kwan@monash.edu

The statistical analysis was conducted by Dr Zhibin Chen at Monash University. Manuscript word count: 2,279

Abstract word count: 249

Title character count: 94

\section{STUDY FUNDING}

This study was supported by an NINDS/Fogarty grant (R21NS069223-01) and Key Research Project of the Chinese Ministry of Science and Technology (2016YFC0904400).

\section{DISCLOSURE}

I. Davagnanam reports no disclosures relevant to the manuscript.

Z. Chen is supported by the Public Health Early Career Fellowship (GNT1156444) from National Health and Medical Research Council (NHMRC) of Australia, and has received research grants from University of Melbourne Early Career Researcher Grant Scheme.

C. Hoskote, D. Ding, B. Yang, Y. Wang, T. Wang, and W. Li report no disclosures relevant to the manuscript.

J. Duncan has received support from Circle Harmony for lecturing.

W. Wang reports no disclosures relevant to the manuscript.

J. Sander has received research grants and honoraria from UCB, Eisai, GW and Zogenix which are involved in the manufacturing of anti-seizure drugs.

P. Kwan is supported by the Medical Research Future Fund Practitioner Fellowship (MRF1136427). He has received research grants from the NHMRC of Australia, the Australian Research Council, the US National Institutes of Health, Hong Kong Research Grants Council, Innovation and Technology Fund, Health and Health Services Research Fund, and Health and Medical Research Fund. He/his institution also received speaker or consultancy fees and/or research grants from Biscayne, Eisai Inc., GW Pharmaceuticals, LivaNova, Novartis, UCB Pharma, and Zynerba. 


\begin{abstract}
Objective

To assess the prevalence of brain MRI abnormalities in people with epilepsy in rural China and to compare this with individuals in the United Kingdom.
\end{abstract}

\title{
Methods
}

Brain MRI scans were obtained in people with epilepsy who participated in a rural community-based program in China between July 2010 and December 2012. Individual epileptogenic lesion types were reviewed and their associations with seizure control examined. The MRI findings were compared with two previous similar studies in the United Kingdom.

\section{Results}

Among the 597 individuals (58\% males; median age 38 years) with MRI scans analyzed, 488 (82\%) had active epilepsy. The MRI was abnormal in 389 subjects (65\%), with potentially epileptogenic lesion in $224(38 \%)$ and non-specific abnormalities in 165 (28\%), and 108 $(18 \%)$ were potentially resectable. The potentially epileptogenic lesions were less frequently detected in children $(<18$ years old, $12 / 68,18 \%)$ than in adults $(212 / 529,40 \% ; p<0.001)$. In people with potentially epileptogenic lesions $67 \%(150 / 224)$ had failed two or more antiseizure medications. They had higher risk of uncontrolled epilepsy than those with normal MRI (risk ratio $[R R]=1.25 ; p<0.001$ ) and those with non-specific abnormality $(\mathrm{RR}=1.15 ; p=0.002)$, after adjusting for age and sex. The diagnostic yield of MRI was similar to that reported in community- and hospital-based studies in the United Kingdom. 


\section{Conclusions}

Over one-third of people with chronic epilepsy in rural China have potentially epileptogenic lesions identifiable on brain MRI, with two-thirds fulfilling the definition of pharmacoresistance. These findings highlight the magnitude of the unmet needs for epilepsy surgery in China. 


\section{INTRODUCTION}

The detection of localized pathology on MRI can decisively impact the diagnostic and management pathways in epilepsy, particularly in people refractory to pharmacological therapy for whom surgery may be a treatment option. Population-based studies on the prevalence of MRI detected lesions have mostly been reported from high-income countries, examining people with new onset ${ }^{1}$ or established epilepsy ${ }^{2,3}$. In comparison, MRI studies have rarely been performed in low-to-middle income countries, where the majority of people with epilepsy live. Given that the incidence and life-time prevalence of epilepsy varies with socio-economic factors ${ }^{4}$, it is possible that the spectrum and frequency of epileptogenic lesions may also vary depending on the socioeconomic and environmental setting of the population. A recent hospital-based study of people with new-onset epilepsy in India identified potentially epileptogenic lesions on MRI in almost half of the cohort, mostly due to infection and inflammation ${ }^{5}$.

Six million people are estimated to have active epilepsy in China ${ }^{6}$ where epilepsy surgery is increasingly performed. ${ }^{7}$ To help estimate the unmet needs for epilepsy surgery in China, we evaluated MRI findings of people with established epilepsy living in the rural communities. The findings were compared with results in previously reported studies from the United Kingdom $^{2,3}$.

\section{METHODS}

\section{Participants}

People with epilepsy aged 2 to 80 years being treated in the National Epilepsy Control Program $^{8}$ in rural areas in four Chinese provinces (Henan, Hebei, Ningxia and Shanxi) were 
recruited between 1 July 2010 and 31 December 2012. People were not included if they had dissociative seizures, alcohol or drug related seizures, progressive or degenerative neurological or systemic disorders, and those whose seizure control or treatment adherence could not be adequately assessed. Pregnant women, people with an intellectual disability unable to provide informed consent, and people in whom MRI is contraindicated, including those with metallic implants or devices and claustrophobia were not included. Seizure outcomes were assessed at recruitment. Seizure freedom was defined as having no seizures for at least the previous 12 months.

\section{Standard Protocol Approvals, Registrations, and Patient Consents}

The study was approved by the Joint Chinese University of Hong Kong-New Territories East Cluster Research Ethics Committee and by the institutional review board of the Beijing Neurosurgical Institute in China. Written informed consent was obtained from all participants or assent by legal guardians when appropriate.

\section{Clinical assessment tools}

As previously described ${ }^{9}$, predesigned epilepsy history and seizure classification questionnaires were used to collect medical history and seizure information from participants or their carers by primary care physicians. The clinical questionnaire consisted of 19 points covering birth, developmental, family, epilepsy, other medical and drug history. The 33question seizure classification questionnaire covered a broad range of seizure semiology for classification in accordance with the updated ILAE terminology. ${ }^{10}$ The questionnaires were developed based on those previously employed for seizure classification ${ }^{11-13}$ and were piloted before deployment. Training and standardization workshops for physicians involved were conducted by senior epilepsy specialists (JWS and PK). 


\section{MRI protocols}

Common brain MRI protocols were developed for the participating epilepsy centers. An MRI (1.5T) brain protocol optimized for the investigation of epilepsy which was feasible in the local settings was established. The scanning protocol included a T1-weighted 3D-volumetric acquisition sequence with $1 \mathrm{~mm}$ isotropic resolution as well as oblique coronal proton density, T2-weighted and fluid-attenuated inversion recovery (FLAIR) sequences ${ }^{14,15}$.

\section{Imaging Analysis}

The MRI acquisitions were anonymized and only identifiable by the designated individual study identification number. These were systematically evaluated on Osirix PACS (Pixmeo, Geneva) ${ }^{16}$ with high-resolution diagnostic monitors by consensus between two senior neuroradiologists (ID and $\mathrm{CH}$ ) blinded to the demographics, seizure semiology and all other clinical details. The scans were evaluated for abnormalities which were potentially epileptogenic as previously classified ${ }^{1}$ as well as all other cranial/intracranial abnormalities.

\section{Statistical analysis}

Epilepsy was considered as active or uncontrolled if the individual had at least one seizure in the preceding 12 months at the time of recruitment. For the purpose of analyzing its association with MRI findings, age was grouped into <18 years, 18-34, 35-64, and 65 years and older. Fisher's exact test was used to compare categorical variables and proportions of potentially epileptogenic lesions between the rural China cohort and UK cohorts. Multivariable generalized linear models were performed to assess the associations between presence of potentially epileptogenic lesion and epilepsy type, age and sex, and epilepsy control and MRI findings. Statistical significance level was set at $p<0.05$. Holm-Bonferroni 
method was applied to correct for multiple comparisons. All statistical tests were performed by using Stata version 15 (StataCorp, College Station, TX).

\section{Data Availability}

Anonymized data will be shared by request from any qualified investigator.

\section{RESULTS}

\section{Demographics and clinical characteristics}

A total of 637 individuals were recruited, of whom 39 were excluded as MRI incompatible and one did not complete the questionnaires. Among those 597 who had an MRI, 344 (58\%) were males. The median age at entry was 38 years (interquartile range [IQR] 27-48), median age of epilepsy onset was 14 years (IQR 6-25) and median duration of epilepsy was 19 years (IQR 11-28). As previously described ${ }^{9}, 525$ (88\%) individuals had focal epilepsy, 54 (9.1\%) had generalized, $1(0.17 \%)$ had combined, and 17 (2.9\%) had unknown classification. At recruitment, $524(88 \%)$ were taking anti-seizure medications (ASMs) and additional 46 (7.7\%) individuals had been previously treated with ASMs (median number of total ASMs tried 2, IQR 1-3). Among all participants, 488 (82\%) individuals had active epilepsy.

\section{MRI findings}

Abnormalities are summarized in Table 1. Of the 597 individuals, MRI was abnormal in 389 (65\%), including potentially epileptogenic lesions in $224(38 \%)$ and non-epileptogenic abnormalities in 165 (28\%). Gliosis or encephalomalacia (109/597, 18\%) was the most common type, which included ulegyria and changes consistent with previous stroke, trauma and surgery. Mesial temporal sclerosis $(83 / 597,14 \%)$ was the second most common epileptogenic lesion found, followed by developmental abnormality (39/597, 6.5\%). Vascular 
anomaly $(9 / 597,1.5 \%)$ and brain tumors $(8 / 597,1.3 \%)$ were relatively uncommon. Twentyseven individuals had dual pathology, including 19 with mesial temporal sclerosis and gliosis/encephalomalacia, 4 with two types of developmental abnormality, 2 with ulegyria and other types of gliosis or encephalomalacia, and 2 with gliosis or encephalomalacia and developmental abnormality.

The vast majority $(219 / 224,98 \%)$ of people with potentially epileptogenic lesions had focal onset seizures, $4(1.8 \%)$ had generalized seizures and $1(0.45 \%)$ had seizures of unknown classification (Table 2). Individuals with focal onset seizures had a significantly higher chance of having a detectable potentially epileptogenic lesion in MRI compared to those who only had generalized onset seizures $(42 \%$ vs. $7.4 \%$; risk ratio $[\mathrm{RR}]=5.54 ; 95 \%$ confidence interval $[\mathrm{C}] 2.14-14.3 ; p<0.001)$ after adjustment for age and sex.

Potentially epileptogenic lesions were less frequently detected in children $(<18$ years old; $12 / 68,18 \%)$ than in adults $(212 / 529,40 \% ; \mathrm{RR}=0.44 ; 95 \%$ CI $0.26-0.74 ; p<0.001)$. Multivariable analysis showed that, compared to children, the risk of detecting potentially epileptogenic lesion were significantly higher in adults aged 18-34 (RR=1.91; 95\% CI 1.123.27; $p=0.018)$ and 35-64 $(\mathrm{RR}=2.05 ; 95 \%$ CI $1.22-3.44 ; p=0.007)$ years, but was not significantly different from those aged 65 years or older $(\mathrm{RR}=1.17$; 95\% CI $0.50-2.76$; $p=0.71)$.

\section{Association between MRI findings and seizure control}

Epilepsy was controlled in 25\% (53/208) of those with normal MRI and 22\% (36/165) in those with non-specific abnormality, but only 9\% (20/224) of those with potentially epileptogenic lesion had full seizure control. People who had potentially epileptogenic lesion 
had significantly higher risk of uncontrolled epilepsy than those who had normal MRI $(\mathrm{RR}=1.25 ; 95 \%$ CI $1.14-1.36 ; p<0.001)$ and those with non-specific abnormality in MRI $(\mathrm{RR}=1.15 ; 95 \%$ CI 1.06-1.26; $p=0.002)$ after adjusted for age and sex. Among the 224 individuals with potentially epileptogenic lesion, 150 (67\%) had active epilepsy despite trials of two or more ASMs.

\section{Comparison with previous similar studies}

Table 3 illustrates the rates of potentially epileptogenic lesions detected on MRI in rural China and two previous studies in United Kingdom using the same scanning protocol. The early study ${ }^{2}$ was community-based and consisted of 174 people with chronic epilepsy who had MRI (1.5T) performed between June 1995 and May 1997. 81/174 (47\%) were males. Their median age was 37 years (range $15-73$ years). $72 \%$ had focal, $25 \%$ generalized, and 3\% combined focal and generalized epilepsy. All had 4 or more-year history of epilepsy with at least 1 seizure during the preceding 12 months. The proportion and individual types of potentially epileptogenic lesions were similar to our cohort. Another hospital-based study ${ }^{3}$ of 804 individuals (87\% focal, 8\% generalized and 5\% unclassified epilepsy) first attendance for an epilepsy MRI scan (1.5T) between May 1995 to June 2004 also reported similar potentially epileptogenic lesion rate $(36 \%)$ and types.

\section{DISCUSSION}

We identified potentially epileptogenic lesions in brain MRI in over one-third of people with epilepsy living in rural China. Gliosis or encephalomalacia accounted for nearly half of the epileptogenic lesions found. The prevalence of mesial temporal sclerosis was also high in the cohort. A number of individuals were found to have various developmental abnormalities. Presence of potentially epileptogenic lesion was associated with focal onset seizures and 
uncontrolled epilepsy. The potentially epileptogenic lesions were less prevalent in children compared to adults, likely because a higher proportion of children would have genetic epilepsies while acquired epilepsies are more common in adults. The diagnostic yield and types of lesions were comparable to findings in the United Kingdom.

Notably, a large proportion of the lesions identified are generally considered as amenable to resective epilepsy surgery, including mesial temporal sclerosis (14\% of the total cohort), vascular malformations (1.5\%), focal cortical dysplasia (1.3\%) and tumors (1.3\%). Combined, these lesions were found in almost a fifth of the participants. These lesions are frequently associated with pharmacoresistance ${ }^{17}$. Indeed, less than $10 \%$ of individuals with potentially epileptogenic lesions had full seizure control in our cohort, and $67 \%$ would be considered to have pharmacoresistant epilepsy having failed two or more $\mathrm{ASMs}^{18}$.

While the mesial temporal sclerosis rate was high in our cohort, it was reported that surgeries for mesial temporal sclerosis in epilepsy centers in western countries has declined ${ }^{19}$. People with mesial temporal sclerosis in our cohort may have been previously missed due to the lack of radiological expertise in Rural China. Epilepsy surgery also has shorter history in China particularly in rural setting. Further, even when hippocampal sclerosis was detected, people might not have had access to further pre-surgical evaluation and subsequent intervention. It is also possible that the incidence of mesial temporal sclerosis developing in rural China is higher than in high-income countries. Improvement in pediatric care has been associated with a decline of new cases of mesial temporal sclerosis over the last 30 years in high income countries. We speculate that these factors might have accounted for the relatively high prevalence of mesial temporal sclerosis detected in this study compared with the declining rate of this pathology in surgical series from western countries. 
These findings have major implications for service provision and highlight the considerable unmet needs in epilepsy management in China, particularly surgical therapy and the required access to specialist neuroimaging. Six million people in China have active epilepsy ${ }^{6}$, for whom neuroimaging with MRI should be an integral part of evaluation. Extrapolating our findings to the rest of the population, it may be estimated that as many as 1 million individuals with epilepsy may have potentially resectable epileptogenic lesions in China. The most common type of such lesions we identified was mesial temporal sclerosis, for which anterior temporal lobectomy could achieve seizure freedom in over $70 \%$ of people $e^{20}$. Identification of these lesions on MRI should, therefore, trigger presurgical evaluation in epilepsy centers, including optimization of drug treatment, video-EEG monitoring and neuropsychological assessment. It is almost certain that only a small fraction of people with these lesions has been evaluated for, and even fewer received surgery ${ }^{21}$.

Meeting this demand would require concerted effort in healthcare resource planning. As the potentially resectable epileptogenic lesions were more prevalent in people with focal seizures than those with generalized seizures, seizure classification may be used to help prioritize the provision of MRI services. Our findings show that this is possible with the use of standardized questionnaires even in the primary care setting.

Two previous studies in United Kingdom using similar protocols also had high proportions of focal epilepsy ( $72 \%$ and $87 \%$ ) comparable to the rural China cohort $(88 \%)$. The three studies also had similar MRI diagnostic yields, detecting similar types of potentially epileptogenic lesions and high proportions of mesial temporal sclerosis. Given that these studies employed 1.5T MRI scanners, with the advent of high-field magnets at 3T and internationally 
recommended imaging protocol ${ }^{22}$, it is likely that the detection rates of lesions reported in these studies represented an under-estimation, and the number of cases who should be considered for pre-surgical evaluation might be even higher.

The study has limitations. There was a bias towards people with convulsive seizures. This might be because those with non-convulsive seizures were less likely to seek medical care in the rural setting. Moreover, most of the individuals in this cohort had chronic and active epilepsy. Therefore, the findings may also not reflect the etiologies of new onset epilepsy, but rather the burden of pathological lesions in people with active or pharmacoresistant epilepsy.

Through a standardized and locally feasible MRI protocol we found a high prevalence of potentially epileptogenic lesions in people with epilepsy in rural China, particularly in those with focal seizures. Careful seizure classification, aided by standardized questionnaire on semiology, may help prioritize the use of MRI in resource limited settings. Our diagnostic yield was comparable to people with chronic epilepsy in high-income countries, suggesting similar burden of pathological lesions in people with active epilepsy despite different socioeconomic settings. With an estimated one million people having potentially resectable epileptogenic lesions, these findings call for urgent actions to address this huge unmet need in epilepsy management in China. 


\section{ACKNOWLEDGEMENT}

This work was partly carried out at UCLH/UCL Comprehensive Biomedical Research

Centre, which receives a proportion of funding from the UK Department of Health's NIHR research Centers funding scheme. JWS receives support from the Dr Marvin Weil Epilepsy Research Fund, the UK Epilepsy Society and Christelijke Vereniging voor de Verpleging van Lijders aan Epilepsie, the Netherlands. 


\section{APPENDIX 1: AUTHORS}

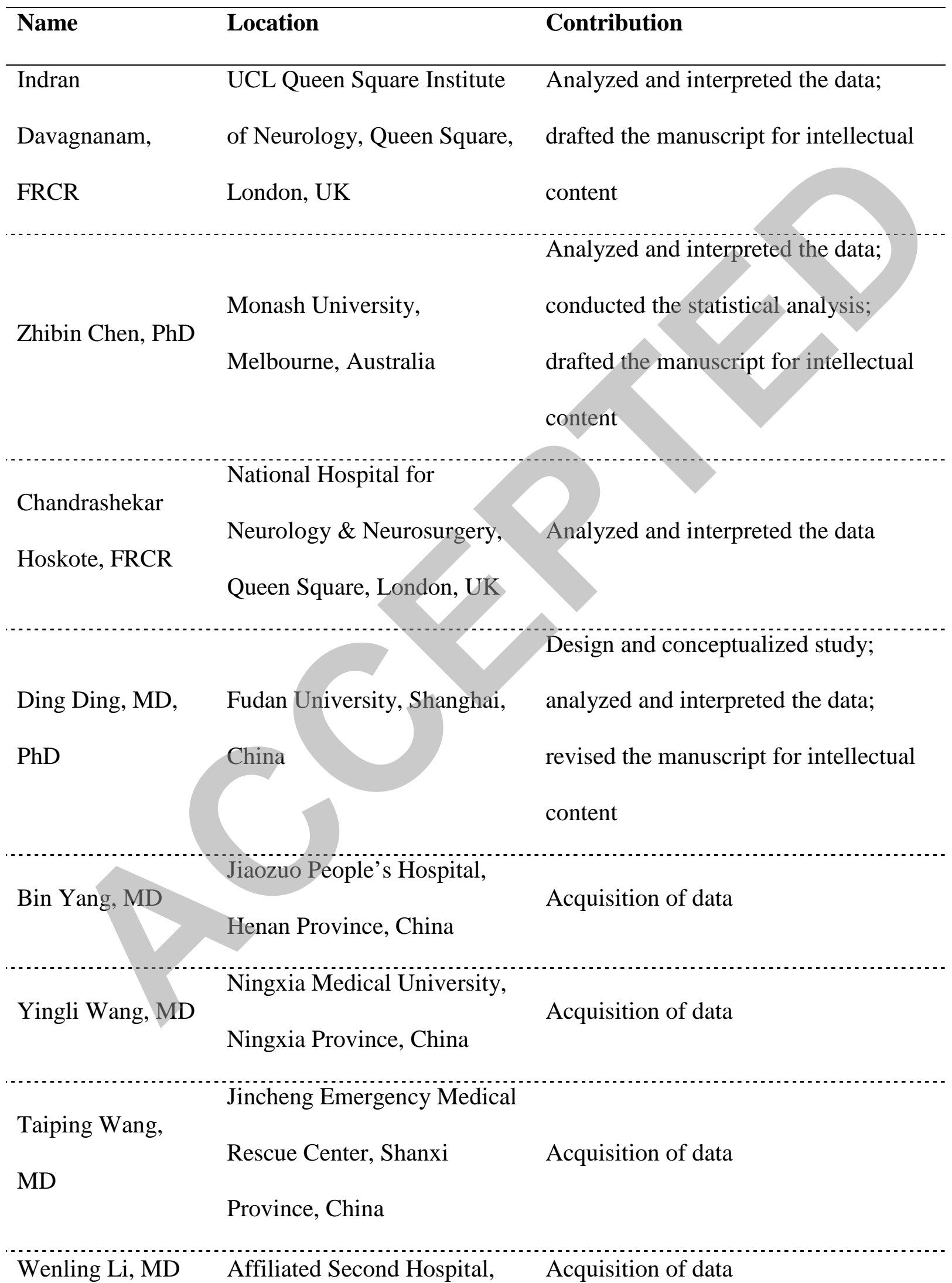


Hebei Medical University,

Hebei Province, China

John S. Duncan,

UCL Queen Square Institute Design of study; interpreted the data;

DM, FRCP

of Neurology, Queen Square, revised the manuscript for intellectual
London, UK
content

Wenzhi Wang, Beijing Neurosurgical

MD Institute, Beijing, China

Acquisition of data; analyzed and interpreted the data; revised the manuscript for intellectual content

Josemir W. UCL Queen Square Institute

Sander, PhD, of Neurology, Queen Square,

Design and conceptualized study;

analyzed and interpreted the data;

FRCP London, UK

revised the manuscript for intellectual

content

Design and conceptualized study;

Patrick Kwan, $\quad$ Monash University, analyzed and interpreted the data;

$\mathrm{PhD}, \mathrm{FRACP}$

Melbourne, Australia

revised the manuscript for intellectual

content 


\section{REFERENCES}

1. Hakami T, McIntosh A, Todaro M, et al. MRI-identified pathology in adults with new-onset seizures. Neurology 2013;81:920-927.

2. Everitt A. The structural basis of the epilepsies: An MRI and epidemiological study. The University of London, 2003. Available at: https://search.proquest.com/docview/1752382883 (accessed 19 January 2020).

3. Winston GP, Micallef C, Kendell BE, et al. The value of repeat neuroimaging for epilepsy at a tertiary referral centre: 16 years of experience. Epilepsy Res 2013;105:349-355.

4. Bell GS, Neligan A, Sander JW. An unknown quantity--the worldwide prevalence of epilepsy. Epilepsia 2014;55:958-962.

5. Ponnatapura J, Vemanna S, Ballal S, Singla A. Utility of Magnetic Resonance Imaging Brain Epilepsy Protocol in New-Onset Seizures: How is it Different in Developing Countries? J Clin Imaging Sci 2018;8:43.

6. Wang WZ, Wu JZ, Wang DS, et al. The prevalence and treatment gap in epilepsy in China: an ILAE/IBE/WHO study. Neurology 2003;60:1544-1545.

7. $\mathrm{Xu} \mathrm{L,} \mathrm{Xu} \mathrm{M.} \mathrm{Epilepsy} \mathrm{surgery} \mathrm{in} \mathrm{China:} \mathrm{past,} \mathrm{present,} \mathrm{and} \mathrm{future.} \mathrm{Eur} \mathrm{J} \mathrm{Neurol}$ 2010;17:189-193.

8. Wang W, Wu J, Dai X, et al. Global campaign against epilepsy: assessment of a demonstration project in rural China. Bull World Health Organ 2008;86:964-969.

9. Wang F, Chen Z, Davagnanam I, et al. Comparing two classification schemes for seizures and epilepsy in rural China. Eur J Neurol 2019;26:422-427.

10. Berg AT, Berkovic SF, Brodie MJ, et al. Revised terminology and concepts for organization of seizures and epilepsies: report of the ILAE Commission on Classification and Terminology, 2005-2009. Epilepsia 2010;51:676-685. 
11. Reutens DC, Howell RA, Gebert KE, Berkovic SF. Validation of a questionnaire for clinical seizure diagnosis. Epilepsia 1992;33:1065-1071.

12. Placencia M, Suarez J, Crespo F, et al. A large-scale study of epilepsy in Ecuador: methodological aspects. Neuroepidemiology 1992;11:74-84.

13. Ottman R, Barker-Cummings C, Leibson CL, Vasoli VM, Hauser WA, Buchhalter JR. Validation of a brief screening instrument for the ascertainment of epilepsy. Epilepsia 2010;51:191-197.

14. Recommendations for neuroimaging of patients with epilepsy. Commission on Neuroimaging of the International League Against Epilepsy. Epilepsia 1997;38:1255-1256.

15. Duncan JS. Neuroimaging of the Epilepsies. Rivista di Neuroradiologia 2004;17:446463.

16. Rosset A, Spadola L, Ratib O. OsiriX: an open-source software for navigating in multidimensional DICOM images. J Digit Imaging 2004;17:205-216.

17. Semah F, Picot MC, Adam C, et al. Is the underlying cause of epilepsy a major prognostic factor for recurrence? Neurology 1998;51:1256-1262.

18. Kwan P, Schachter SC, Brodie MJ. Drug-resistant epilepsy. The New England journal of medicine 2011;365:919-926.

19. Jehi L, Friedman D, Carlson C, et al. The evolution of epilepsy surgery between 1991 and 2011 in nine major epilepsy centers across the United States, Germany, and Australia. Epilepsia 2015;56:1526-1533.

20. West S, Nevitt SJ, Cotton J, et al. Surgery for epilepsy. Cochrane Database Syst Rev 2019.

21. Xiao B, Huang ZL, Zhang H, et al. Aetiology of epilepsy in surgically treated patients in China. Seizure : the journal of the British Epilepsy Association 2004;13:322-327. 
22. Bernasconi A, Cendes F, Theodore WH, et al. Recommendations for the use of structural magnetic resonance imaging in the care of patients with epilepsy: A consensus report from the International League Against Epilepsy Neuroimaging Task Force. Epilepsia 2019;60:1054-1068. 


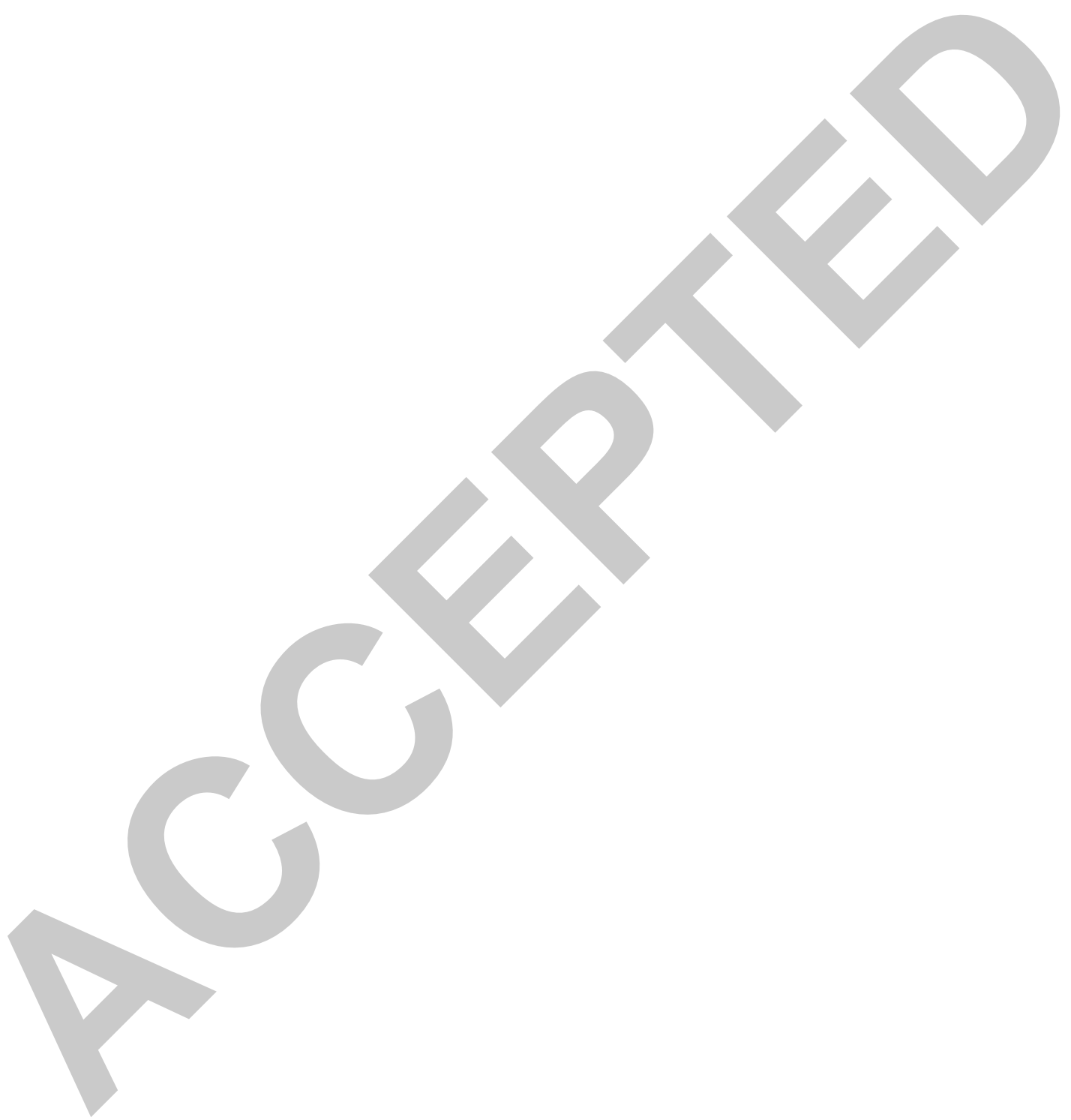

Copyright (C) 2020 American Academy of Neurology20 nauthorized reproduction of this article is prohibited 


\section{TABLES}

Table 1. Summary of MRI findings in the 597 people with epilepsy in rural China

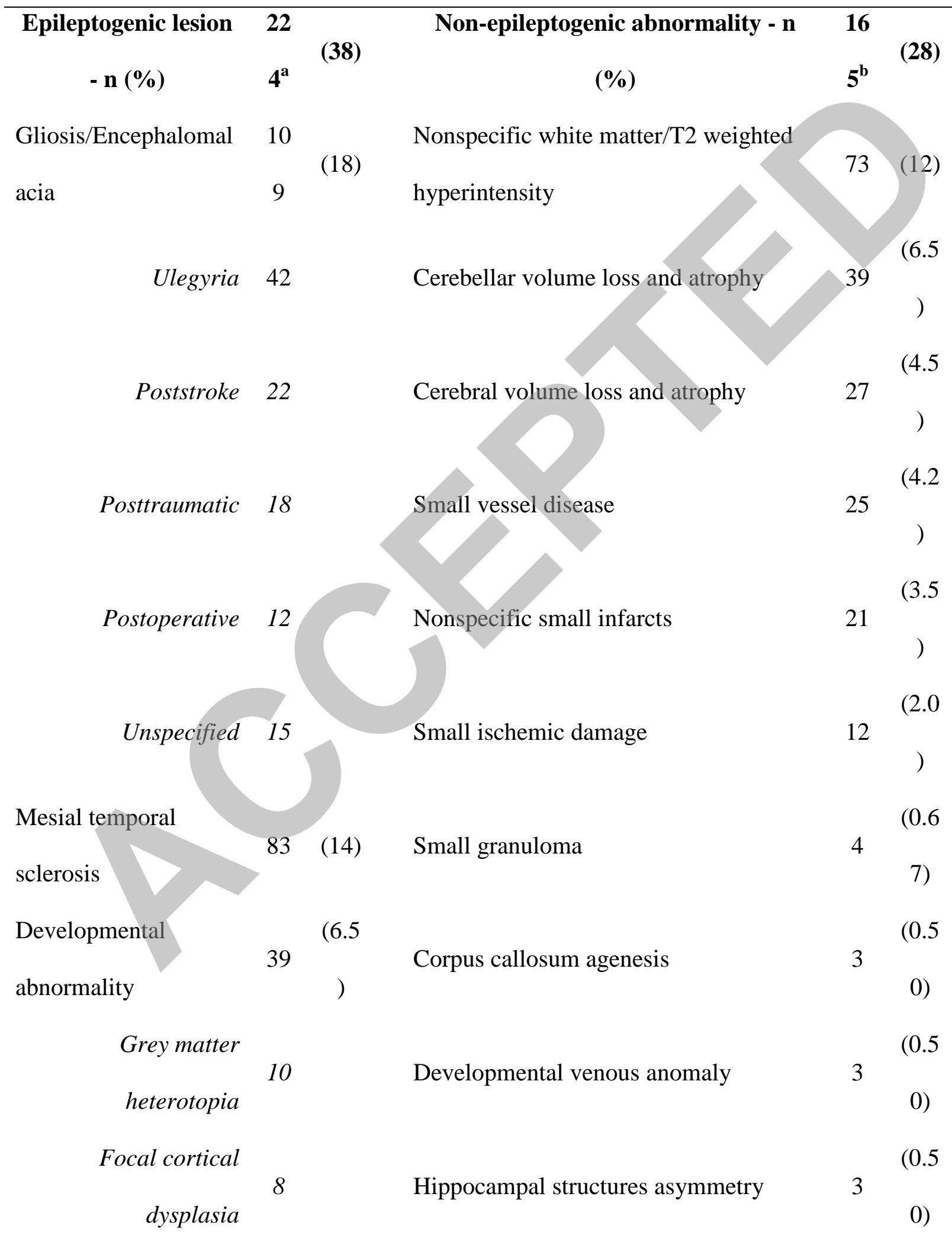


Polymicrogyria 8

Chiari I malformation

$(0.1$

1

7)

$(0.1$

Arachnoid cyst 4

Choroidal fissure cyst

1

7)

$(0.1$

Tuberous sclerosis 4 Cystic lesion

Nodular heterotopia $1 \quad$ Leukodystrophy

$(0.1$

Local dystrophy

Band heterotopia 2

Subdural cystic

hygroma

Nonspecific nodule

1

Perinatal ischemic

Rathke's cleft cyst
7)

1

$(0.1$

1

$(0.1$

1

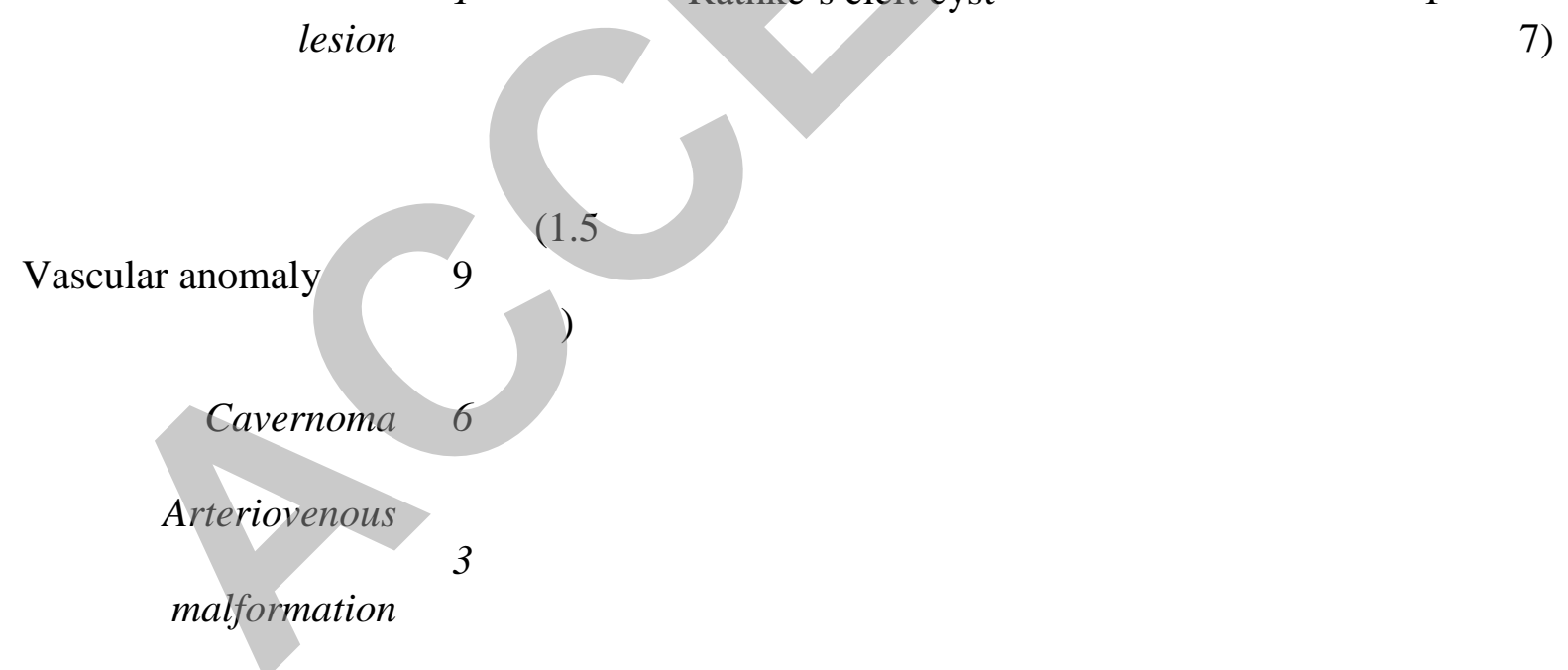

$(1.3$

20

$8 \quad)$

No abnormality detected - n (\%)

(35)

8

\section{Glioma 5}

Nonspecific tumor 3 
Neurocysticercosis

$(0.3$

2

4)

Granulomatous

$(0.1$

inflammation

1

7)

Total percentage totals to slightly more than $100 \%$ owing to rounding.

a. Twenty-seven people had dual pathology.

b. Among people without epileptogenic lesions, 38 had dual abnormalities and seven had three types of nonepileptogenic abnormalities. 
Table 2. Frequency of MRI findings according to seizure types and epilepsy classifications

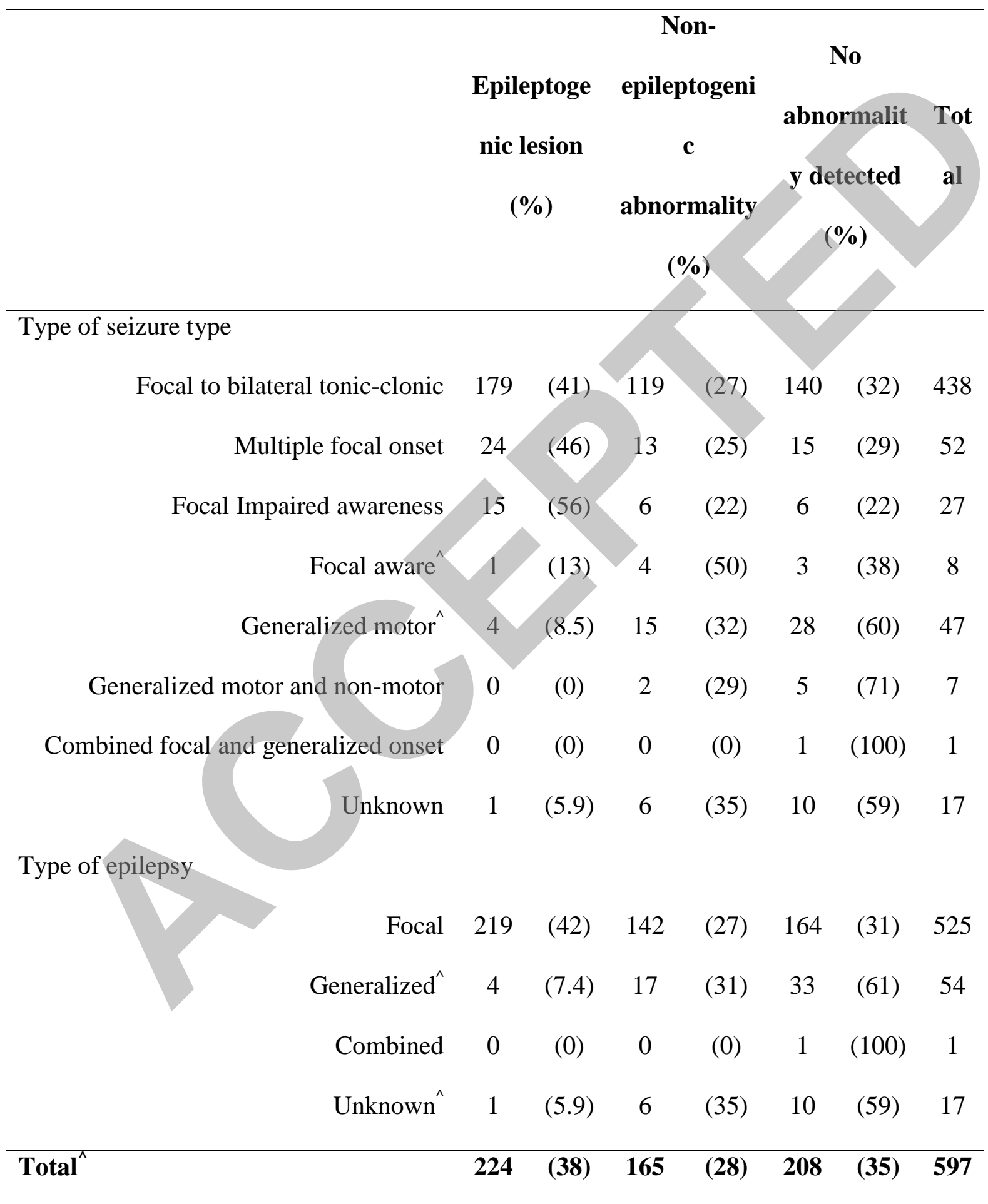

$\wedge$ Percentages total to slightly more or less than $100 \%$ owing to rounding. 
Table 3. Potentially epileptogenic lesions detected on MRI in people with epilepsy in studies in rural China and United Kingdom

\begin{tabular}{|c|c|c|c|c|c|c|c|c|c|c|c|c|c|c|}
\hline & \multicolumn{2}{|c|}{$\begin{array}{c}\text { Rural China } \\
\qquad(\mathrm{N}=597)\end{array}$} & \multicolumn{6}{|c|}{ UK Community-based (N=174) } & \multicolumn{6}{|c|}{ UK Hospital-based $(\mathrm{N}=804)$} \\
\hline & $\mathbf{n}$ & $(\%)$ & $\mathbf{n}$ & $(\%)$ & $\mathbf{R R}$ & $(95 \% \mathrm{CI})$ & value & & & & $\mathbf{R R}$ & $(95 \% \mathrm{CI})$ & value & $p$-value \\
\hline $\begin{array}{l}\text { People with potentially } \\
\text { epileptogenic lesion }^{\mathrm{a}}\end{array}$ & 224 & (38) & $\leq 71^{b}$ & $(\leq 41)$ & $0.92^{c}$ & $(0.75-1.13)$ & 0.4 & 0.96 & 293 & (36) & 1.03 & $(0.90-1.18)$ & 0.70 & 0.70 \\
\hline Gliosis/Encephalomalacia & 109 & (18) & 36 & $(21)$ & 0.88 & $(0.63-1.2$ & 0.51 & $>0.99$ & 145 & $(18)$ & 1.01 & $(0.81-1.27)$ & 0.94 & 0.94 \\
\hline Mesial temporal sclerosis & 83 & (14) & 29 & (17) & 0.83 & $(0.57-1.2$ & 0.39 & 0.39 & 88 & $(11)$ & 1.27 & $(0.96-1.68)$ & 0.10 & 0.20 \\
\hline $\begin{array}{l}\text { Developmental } \\
\text { abnormality }\end{array}$ & 39 & $(6.5)$ & 4 & $(2.3)$ & 2.84 & $(1.03-7.84)$ & 0.037 & 0.074 & 42 & $(5.2)$ & 1.25 & $(0.82-1.91)$ & 0.30 & 0.30 \\
\hline Vascular anomaly & 9 & $(1.5)$ & 0 & $(0)$ & NA & NA & 0.22 & 0.44 & 9 & (1.1) & 1.35 & $(0.54-3.37)$ & 0.63 & 0.63 \\
\hline Tumors & 8 & $(1.3)$ & 2 & (1.1) & 1.17 & $(0.25-5.44)$ & $>0.99$ & $>0.99$ & 9 & (1.1) & 1.20 & $(0.46-3.08)$ & 0.81 & $>0.99$ \\
\hline Neurocysticercosis & 2 & $(0.34)$ & 0 & (0) & NA & NA & $>0.99$ & $>0.99$ & 0 & $(0)$ & NA & NA & 0.18 & 0.36 \\
\hline $\begin{array}{l}\text { Granulomatous } \\
\text { inflammation }\end{array}$ & 1 & $(0.17)$ & 0 & $(0)$ & NA & NA & $>0.99$ & $>0.99$ & 0 & $(0)$ & NA & NA & 0.43 & 0.85 \\
\hline
\end{tabular}




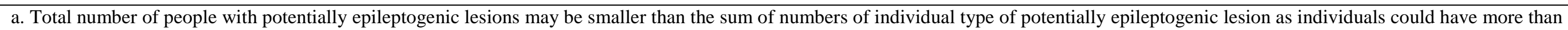
one type of potentially epileptogenic lesion.

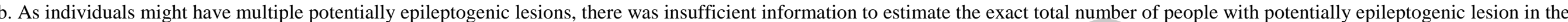

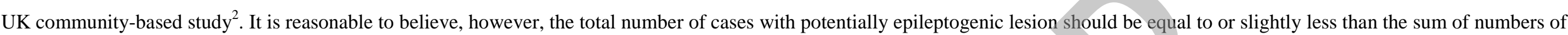
individual types of potentially epileptogenic lesion.

c. The sum of numbers of individual types of potentially epileptogenic lesion was used in the comparison analysis.

CI, confidence interval; NA, not applicable; RR, risk ratio 